\title{
Male Breast Cancer: Surgical and Genetic Features and a Multidisciplinary Management Strategy
}

\author{
Francesca Pellini $^{a}$ Eleonora Granuzzo ${ }^{a}$ Silvia Urbani ${ }^{a}$ Sara Mirandola ${ }^{a}$ \\ Marina Caldana ${ }^{a}$ Davide Lombardi $^{a}$ Elena Fiorio $^{b}$ Marta Mandaràc \\ Giovanni Paolo Pollini ${ }^{a}$ \\ ${ }^{a}$ Complex Operative Unit of Breast Surgery, Breast Unit, Oncologic Surgery Department, AOUI, Verona, Italy; \\ ${ }^{b}$ Complex Operative Unit of Oncology, Breast Unit, Oncologic Surgery Department, AOUI, Verona, Italy; ${ }^{c}$ Unit of \\ Oncology, San Bonifacio Hospital-ULSS 9, Verona, Italy
}

\section{Keywords}

Male breast cancer $\cdot B R C A 1 / 2 \cdot$ Prostate cancer

\begin{abstract}
Background: Male breast cancer $(\mathrm{MBC})$ is a rare disease with a rising incidence trend. The major risk factors related to $M B C$ are a positive family history of breast cancer (BC) and BRCA1/2 mutations, which indicate a relevant genetic role. Methods: In this retrospective series, we enrolled 69 male patients presenting with male breast cancer (MBC) between 01/01/1992 and 31/12/2018, and 26 high-risk not-affected men presenting between 01/01/2016 and 31/12/2018. Participants' electronic clinical records were reviewed. Patients' data reported age at diagnosis, tumor characteristics, therapeutic management, and BRCA1/2 status as well as a family history of breast, ovarian, or prostate cancer (PCa) in first-degree relatives. $\boldsymbol{R} \boldsymbol{e}$ sults: We analyzed 69 MBC patients. Median age was 64 years. The majority of tumors diagnosed were of an early TNM stage. The most frequent histological subtype was invasive ductal carcinoma (76.7\%). Hormone receptors were positive in $>90 \%$ of MBC cases. Nearly all patients underwent modified radical mastectomy or total mastectomy. Adjuvant endocrine therapy was delivered in 59.4\%. Among MBC-affected patients, we recorded a high percentage of a positive family history of $\mathrm{BC}$. Mutational analysis for the BRCA1/2 genes was performed in $17 \mathrm{MBC}$ patients; $11.8 \%$ were carriers of $B R C A 2$ pathogenic mutations. Among 26 healthy highrisk subjects included in this case series, 4 were $B R C A 1$ muta-
\end{abstract}

tion carriers and 9 were BRCA2 mutation carriers. Discussion: We evaluated the distribution of clinicopathological characteristics in MBC subjects and assessed the frequency of mutations in the BRCA genes in affected patients and healthy high-risk subjects, with the aim of proposing a surveillance program for BC and PCa.

(c) 2019 S. Karger AG, Basel

\section{Introduction}

Male breast cancer (MBC) is a rare disease that accounts for about $0.1 \%$ of all cancers in men [1] and about $1 \%$ of all cases of breast cancer (BC) [2]. MBC incidence is estimated at $<1$ per 100,000 men-years [3] and has increased by about $26 \%$ over the past 25 years [2]. Several risk factors are involved in MBC etiology: hormonal imbalance, occupational and environmental exposure, and genetic factors [4]. A positive family history of $\mathrm{BC}$ and $B R C A 1 / 2$ mutations plays a relevant role in $\mathrm{MBC}$ pathogenesis [4].

Different studies have reported that 15-20\% of male patients with $\mathrm{BC}$ have a family history of $\mathrm{BC}$, a higher percentage than observed in women with BC (7\%) [5-7]. Men with a positive first-degree family history have a 2.0fold increased risk, but this increases to $>10.0$-fold if the number of affected first-degree relatives is $\geq 2$ and if they were diagnosed at a younger age $[2,5]$. 
Moreover, up to $10 \%$ of all MBCs are due to germ-line mutations in the high-penetrance BRCA2 (more frequently recorded) and $B R C A 1$ genes. The estimated lifetime risk of developing $\mathrm{MBC}$ for $B R C A 1$ mutation carriers is in the range of $1-5$ and $5-10 \%$ for $B R C A 2$ mutation carriers, compared with a risk of $0.1 \%$ in the general population [8]. The American Society of Clinical Oncology (ASCO) indeed recommends that genetic counseling and testing should be offered to all men with breast cancer, regardless of their family history [2].

A significantly reduced survival rate is registered in male patients carrying BRCA 1/2 mutations, in line with a younger age at diagnosis, a higher grade and stage, an increased incidence of a second primary tumor at other sites in the body (e.g., the prostate, stomach, colon, pancreas, and skin [melanoma and nonmelanoma]) [5, 8]. Retrospective studies have demonstrated a higher risk of developing mostly prostate cancer (PCa) in BRCA1/2 mutation carriers, and $B R C A 2$ pathogenic mutations lead to PCa earlier and with a more aggressive phenotype [9].

In this retrospective study, we report the results of our observational analysis of the clinical features and treatment of MBC patients, and the family history and $B R C A$ status of patients and healthy high-risk men examined at the Units of Breast Surgery and Oncology, AOUI, and the Unit of Oncology, San Bonifacio Hospital, Verona.

\section{Materials and Methods}

This is a retrospective analysis of all male patients presenting with MBC between 01/01/1992 and 31/12/2018, and high-risk notaffected men presenting between 01/01/2016 and 31/12/2018, at the Units of Breast Surgery and Oncology, AOUI, and the Unit of Oncology, San Bonifacio Hospital, Verona. We enrolled male subjects with a personal or family history of $\mathrm{MBC}$, a family member $B R C A 1 / 2$ gene mutation carrier, a close family member diagnosed with female BC (FBC) at the age of $\leq 45$ years, a close family member diagnosed with bilateral $\mathrm{BC}, \geq 3$ close family members diagnosed with $\mathrm{BC}$ at any age, a close family member diagnosed with pancreatic cancer or metastatic PCa at any age, or with a family history of ovarian cancer, pancreatic cancer, aggressive $\mathrm{PCa}$, or metastatic PCa. Female subjects, male subjects affected by benign breast tumors, and healthy men with a family history not suggestive of genetic mutations linked to BC were excluded.

Clinicopathological and genetic data were obtained from our Breast Unit Database (Gecos, Cartelle2000), which has collected patients' and high-risk subjects' clinical records since January 1992. No additional tests were performed.

We recorded patients' age at diagnosis, tumor characteristics (the affected side, multifocality, stage, histotype, grading, hormonal and human epidermal growth factor receptor 2 [HER2] status, and Ki-67 expression), treatment (type and date of surgery, surgical axillary management, endocrine therapy, chemotherapy, and radiotherapy), a family history of $\mathrm{BC}$, ovarian cancer, or $\mathrm{PCa}$ in first-degree relatives, and $B R C A 1 / 2$ mutation status (if available). For high-risk not-affected men, we recorded a family history of $\mathrm{BC}$, ovarian cancer, or PCa in first-degree relatives, and $B R C A 1 / 2$ mutation status.

MBC: Surgical and Genetic Features and

Multidisciplinary Management
Data regarding histological subtype, histologic or Nottingham grade, estrogen receptor (ER), progesterone receptor (PgR), Ki-67, and HER2 status of BC were collected from the reports of immunohistochemical analysis of sections of formalin-fixed, paraffinembedded mammary tumor blocks. Grading status was carried out according to the modified Bloom-Richardson-Elston grading (BRE) system (also called the Nottingham system) [10]. Ki-67 expression, ER, and PgR were reported as percentages according to international guidelines [11], and ER and PgR positivity was defined as $>1 \%$ of immunoreactive tumor cell nuclei. HER2 status was established by immunohistochemistry (IHC), positivity was settled as a score of $3+$ and negativity as a score of 0 and $1+$ or by fluorescent in situ hybridization (FISH) analysis in ambiguous cases (IHC score of 2+).

In January 2016, a genetic counseling program was established at our center. Patients diagnosed with MBC and high-risk not-affected men were assessed by an expert geneticist specifically dedicated to the study of hereditary BC, ovarian cancer, and PCa. During the ambulatory interview, the geneticist drew genealogical family trees based on participants' family histories. A BRCA test was performed on a blood sample collected at the Breast Unit and sent to the Medical Laboratory Department, where it was validated by fluorimetry technique and multiplex polymerase chain reaction (PCR). BRCA 1 and BRCA2 mutations were classified according to their potential functional effect as recorded in the Breast Cancer Information Core (BIC) database [12] as: class 5, pathogenic; class 4 , likely pathogenic; class 3 , uncertain; class 2 , likely not pathogenic; class 1, not pathogenic.

All collected data were recorded on a Microsoft Office Excel spreadsheet, protected by password, and accessible only to Breast Unit members directly involved in this study. Continuous variables are expressed as medians. Categorial variables are expressed as numbers and percentages. All statistical analyses were performed with Microsoft Office Excel v97 (2003).

\section{Results}

The overall number of subjects was $95 ; 69$ were affected by $\mathrm{MBC}$ and 26 were high-risk not-affected men. At the Units of Breast Surgery and Oncology, AOUI, and the Unit of Oncology, San Bonifacio Hospital, Verona, 52 patients were diagnosed with MBC between January 1992 and December 2015. Forty-three male subjects out of these 95 were involved in the genetic examination between January 2016 and December 2018: 17 patients affected by $\mathrm{MBC}$ and 26 healthy high-risk men.

\section{Clinicopathological Features and Treatment}

Sixty-nine patients received diagnosis and/or treatment for MBC at our center. Clinicopathological features, therapeutic strategies, data regarding family history, and $B R C A$ genes mutational status are showed in Table 1. Age at $\mathrm{MBC}$ diagnosis ranged between 24 and 98 years (median 64.0 years). Most patients $(87 \%)$ were $>50$ years old and the rest were younger (13\%) (Fig. 1).

Right-sided tumors accounted for 58\%. Multifocal breast tumors stood for $5.8 \%$, but no synchronous bilateral $\mathrm{BC}(\mathrm{SBBC})$ was registered. The majority of patients 
Table 1. Clinicopathological features of $69 \mathrm{MBC}$ patients

\begin{tabular}{|c|c|}
\hline Age at diagnosis, years & 64.0 \\
\hline Family history & $23(33)$ \\
\hline \multicolumn{2}{|l|}{ Breast cancer } \\
\hline Ovarian cancer & $1(1.4)$ \\
\hline Prostatic cancer & $5(7.2)$ \\
\hline Pancreatic cancer & $2(2.9)$ \\
\hline \multicolumn{2}{|l|}{ Affected side } \\
\hline Left & $29(42)$ \\
\hline Right & $40(58)$ \\
\hline Both & $0(0)$ \\
\hline \multicolumn{2}{|l|}{ Tumor stage } \\
\hline 0 & $8(11.6)$ \\
\hline I & $23(33.3)$ \\
\hline II & $19(27.5)$ \\
\hline III & $16(23.2)$ \\
\hline IV & $3(4.3)$ \\
\hline \multicolumn{2}{|l|}{ Histotype } \\
\hline Ductal component & $64(92.7)$ \\
\hline Lobular & $1(1.4)$ \\
\hline Other & $4(5.8)$ \\
\hline \multicolumn{2}{|l|}{ Hormone receptor status } \\
\hline ER-positive & $57 / 59(96.6)$ \\
\hline PgR-positive & $53 / 56(94.6)$ \\
\hline HER2-negative & $20 / 42(47.6)$ \\
\hline \multicolumn{2}{|l|}{ Node status } \\
\hline N0 & $32(46.4)$ \\
\hline N1 & $18(26)$ \\
\hline $\mathrm{N} 2$ & $9(13)$ \\
\hline N3 & $1(1.4)$ \\
\hline Unknown & $9(13)$ \\
\hline Ki-67 high-level (cutoff 20\%) & $22 / 59(37.3)$ \\
\hline \multicolumn{2}{|l|}{ Surgery management } \\
\hline Mastectomy & $66(95.6)$ \\
\hline Breast-conserving surgery & $3(4.4)$ \\
\hline Relapse & $10 / 63(15.9)$ \\
\hline \multicolumn{2}{|l|}{$B R C A$ test } \\
\hline No & $52(75.4)$ \\
\hline Yes & $17(24.6)$ \\
\hline \multicolumn{2}{|l|}{$B R C A$ mutation $(n=17)$} \\
\hline$B R C A 1$ & $0(0)$ \\
\hline$B R C A 2$ & $2(11.8)$ \\
\hline Unknown variant sequence & $7(41.2)$ \\
\hline Negative & $8(47)$ \\
\hline
\end{tabular}

Values express $n$ (\%), unless otherwise indicated. ER, estrogen receptor; PgR, progesterone receptor.

(54\%) had BC of an early TNM stage: $11.6 \%$ had stage 0 , $33.3 \%$ had stage I, and $27.5 \%$ had stage II (most had axillary lymph node involvement). The rest were diagnosed as late-stage: $23.2 \%$ with stage III, and $4.4 \%$ with metastatic disease (stage IV).

The most common MBC histological subtype was invasive ductal carcinoma (IDC) in $76.7 \%$ of the patients; $11.6 \%$ had ductal carcinoma in situ (DCIS), $5.7 \%$ had papillary carcinoma, $1.5 \%$ had invasive mucinous carcinoma, $1.5 \%$ had secretory carcinoma, $1.5 \%$ had inflam-

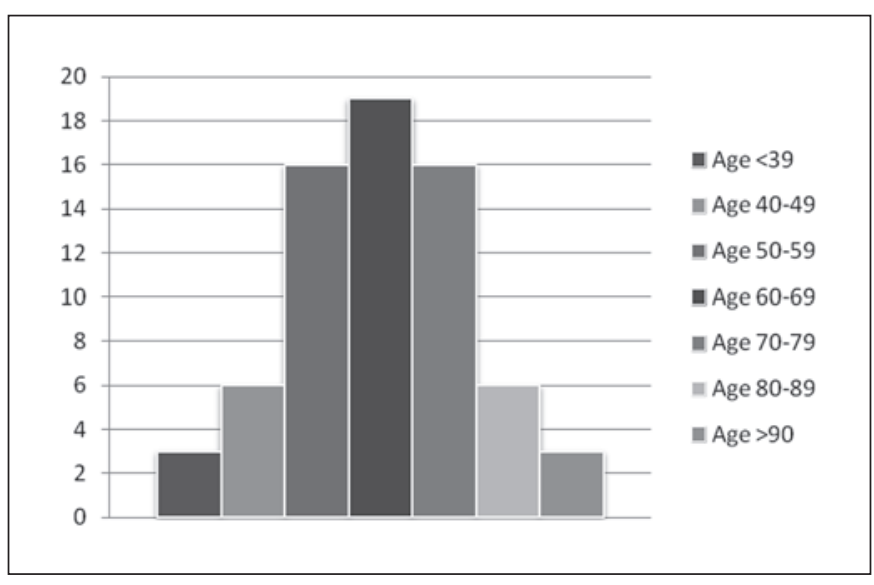

Fig. 1. Age distribution in patients with $\mathrm{MBC}$.

matory $\mathrm{BC}$, and $1.5 \%$ had invasive lobular carcinoma. Histologic or Nottingham grade data were available for 57 patients; 39 (68.4\%) were grade 2.

ER status was available for 59 patients: $96.6 \%$ ER-positive and 3.4\% ER-negative. PgR status was available for 56 patients: 94.6\% PgR-positive and 5.4\% PgR-negative. HER2 status was known in 42 patients and was positive in $52.4 \%$ cases. Ki-67 expression was assessed in 59 samples: $62.7 \%$ had $<20 \%$ positive cells and $37.3 \%$ had $\geq 20 \%$ positive cells (corresponding with high Ki-67 expression).

Modified radical mastectomy or total mastectomy was performed on $95.6 \%$ patients while $4.4 \%$ underwent corebiopsy only.

Most patients (87\%) sustained axillary nodal procedures: $16.7 \%$ had sentinel lymph node biopsy (SLNB) and no pathologically positive lymph nodes, $3.3 \%$ had SLNB followed by axillary lymph node dissection (ALND) following documented pathologically positive lymph nodes, and $80 \%$ had ALND without previous SLNB.

The systemic medical treatment consisting of adjuvant endocrine therapy was administered in $59.4 \%$ patients, nearly all with tamoxifen. Adjuvant chemotherapy was prescribed to $27.5 \%$ patients and $18.8 \%$ patients received chemotherapy and endocrine therapy in a sequential manner. Neoadjuvant chemotherapy was administered in $1.4 \%$. Adjuvant trastuzumab was administered to $5.8 \%$ patients. Postmastectomy adjuvant radiation therapy was delivered to $13 \%$ and palliative care to $1.4 \%$.

\section{Genetic Features}

Male $\mathrm{BC}$ predisposing factors include a family history of $\mathrm{BC}$ and $B R C A$ pathogenic mutations. Among MBCaffected patients, $34.8 \%$ were recorded (a high percentage) as having a positive family history of BC. Furthermore, a positive family history of cancer at other sites was investigated and revealed: $7.2 \%$ PCa, $2.9 \%$ pancreatic 
Table 2. Clinicopathological features of $B R C A 2$ mutation carriers

\begin{tabular}{lll}
\hline Characteristics & BRCA-positive & \\
\cline { 2 - 3 } & patient 1 & patient 2 \\
\hline Age at diagnosis & 66 years & 90 years \\
Family history & 2 sisters FBC, & mother FBC, \\
& 2 aunts FBC, & niece FBC \\
Affected side & 1 cousin FBC & left \\
Stage & left & IIIA \\
Histotype & IA & IDC \\
HR status & IDC + DCIS & ER-positive $(80 \%)$ \\
& ER-positive (90\%) & PgR-positive $(80 \%)$ \\
Node status, positive & PgR-positive (80\%) & NX \\
HER2-positive & No & negative \\
Ki-67 high-level (cutoff 20\%) & negative & $25 \%$ \\
Surgery & $15 \%$ & core biopsy \\
Radiotherapy & modified radical mastectomy & No \\
Chemotherapy & No & No \\
Endocrine therapy & No & Yes (tamoxifen) \\
Trastuzumab & Yes (tamoxifen) & No \\
Relapse & No & No \\
\hline
\end{tabular}

ER, estrogen receptor; HR, hormone receptor; PgR, progesterone receptor; IDC, invasive ductal carcinoma; DCIS, ductal carcinoma in situ.

cancer, $7.2 \%$ lung cancer, $5.8 \%$ stomach cancer, $1.5 \%$ ovarian cancer, and $2.9 \%$ colon cancer.

From January 2016 to December 2018, 43 men (17 $\mathrm{MBC}$ patients and 26 high-risk not-affected men) underwent genetic counseling and testing at our center. Mutational analysis for $B R C A 1 / 2$ genes was performed in 17 MBC patients: $11.8 \%$ were carriers of $B R C A 2$ pathogenic mutations, $41.2 \%$ had $B R C A 1 / 2$ benign mutations or an unknown variant sequence, and $47 \%$ had wild-type $B R C A$ genes. Clinicopathological features of MBC BRCA2 mutation carriers are shown in Table 2. Among 26 high-risk men who were involved in BRCA testing: $34.6 \%$ were $B R C A 2$ mutation carriers, $15.4 \%$ were $B R C A 1$ mutation carriers, and $50 \%$ had wild-type $B R C A$ genes. Most of them $(77 \%)$ had a positive family history of breast cancer, $19.2 \%$ of BC and PCa, $19.2 \%$ of ovarian cancer, and 3.8\% of pancreatic cancer.

\section{Discussion}

In this retrospective study, we report characteristics of 69 patients diagnosed with $\mathrm{MBC}$ and analyzed their clinicopathological features, surgical and oncological treatment, and associated genetic risk factors. We also evaluated 26 healthy subjects at a high risk of developing $\mathrm{MBC}$, who underwent genetic counseling and $B R C A$ gene mutation testing.
Median age at diagnosis in those presenting with $\mathrm{MBC}$ was 64 years, and most patients $(87 \%)$ were $>50$ years. This finding might be explained by a lack of awareness of the early signs of MBC and of specific screening programs, in line with previous literature [13].

In our sample, we noted a predominance of right-sided BC ( $58 \%$ vs. $42 \%$ left-sided) and no bilateral BC was detected.

As expected, the most frequent histological subtype was IDC, and just 1 patient was diagnosed with a lobular carcinoma. This confirms the rarity of the lobular histotype of BC in men [2], in line with results from other retrospective case series carried out in Italian men by Gargiulo et al. [13] and Masci et al. [14]. MBC is described as presenting with a higher incidence of nodal metastases and advanced stage at presentation [2], due to a consistent diagnostic delay. Our findings are in line with those of Gargiulo et al. [13], who reported a heterogeneous distribution of tumor stage at diagnosis, with a predominance of early stage tumors.

We found a high rate of hormone-receptor positivity, coinciding with previous observations [4]. Indeed, among those with available data, $96.6 \%$ were ER-positive and $94.6 \%$ were PgR-positive. We also registered $52.4 \%$ of patients as HER2-positive, higher than what is reported in the literature. Indeed, data regarding HER2 status in $\mathrm{MBC}$ are conflicting, with HER2 overexpression rates ranging from 3 to $40 \%[13,15]$. We recorded a single case 


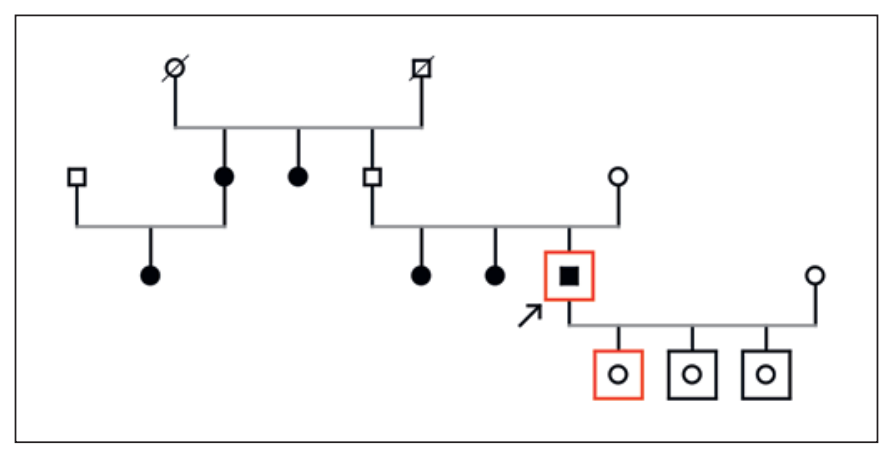

Fig. 2. Patient 1 genealogical tree. Squares, males; circles, females; black filling, breast cancer; white filling, unaffected; black frame, $B R C A 1 / 2$ wild-type; red frame, $B R C A 2$ mutation carrier.

of triple-negative $\mathrm{BC}$ in a man aged 57 years, who was diagnosed as having an advanced stage (IIIB) and experienced a local recurrence of disease. We confirm what was previously reported about a low frequency of triple-negative BCs among men [2].

The most frequently performed surgical procedures for locoregional treatment of $\mathrm{MBC}$ were modified radical mastectomy (MRM) and total mastectomy, performed in 66 of our patients $(95.6 \%)$, consistent with a previous study $[13,16]$.This approach was probably preferred given the anatomic characteristics of male breast tissue, the limited surgical sequelae, and the better cosmetic outcome when compared to other procedures [5]. Most of our patients $(87 \%)$ underwent axillary dissection, as suggested in the literature $[13,16]$. Only 13 patients in our case series underwent SLNB; this is not surprising, given the given the relatively recent introduction of this technique into clinical practice for MBC management [17].

Regarding postsurgical management, postoperative radiotherapy was delivered to 10 patients in our retrospective study ( $13 \%$ of those with available data). Although there are no controlled clinical trials, numerous studies show that radiotherapy reduces the frequency of locoregional relapse [18] and is administered as adjuvant therapy, also in men.

Given the high rate of hormone receptor-positive MBC observed in our sample, it is understandable that most of our patients had adjuvant hormone therapy (59.4\%). Adjuvant hormone treatment with tamoxifen is reported to significantly improve disease-free and overall survival, thereby representing the standard of care [19]. In our study, tamoxifen was the most frequent adjuvant hormone therapy. The role of chemotherapy in $\mathrm{MBC}$ is not well defined and only CMF (cyclophosphamide, metothrexate, 5-fluorouracil) has been prospectively evaluated in the adjuvant setting [19]. The CMF regimen was adopted in about a half of our retrospective study chemotherapy-treated cases. Overall, adjuvant chemo-

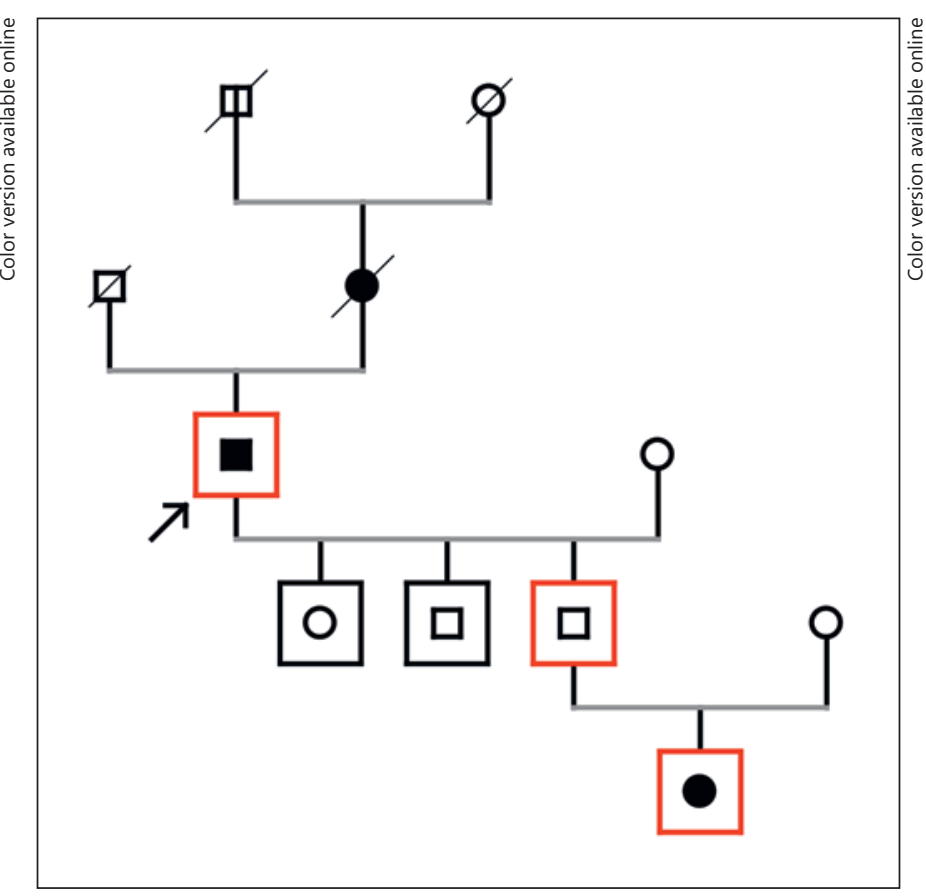

Fig. 3. Patient 2 genealogical tree. Squares, males; circles, females; black filling, breast cancer; white filling, unaffected; black frame, $B R C A 1 / 2$ wild-type; red frame, $B R C A 2$ mutation carrier.

therapy was administered to $46.3 \%$ of patients in our case series (among those with available data). A patient's evaluation before delivery of adjuvant chemotherapy was performed considering different clinical and pathological features such as tumor stage, ER/PgR and Ki-67 level, HER2 status, age and comorbidities, as is commonly done in female BC patients, to select those who may benefit from systemic treatment. The high percentage of patients undergoing chemotherapy could be due to different factors. Nearly $50 \%$ of patients had nodal positive disease, $37.3 \%$ of patients were found to have high levels of Ki-67, and no patients presented age-related comorbidities contraindicating chemotherapy.

A relevant role in $\mathrm{MBC}$ pathogenesis is played by genetic risk factors. Many studies show that $15-20 \%$ of male patients with $\mathrm{BC}$ have a family history of $\mathrm{BC}$, a higher percentage than observed in women with $\mathrm{BC}(7 \%)$ [5-7]. Accordingly, among our patients, we recorded a high percentage of a positive family history of BC: $34.8 \%$.

A correlation between positive family history of pancreatic and gastric cancer and an increased risk of developing MBC has also been described [5, 8]. We observed 6 cases of a positive family history of pancreatic or gastric cancer. This finding is in line with previous literature [5, $8,13]$.

Among genetic risk factors, $B R C A 1 / 2$ gene mutations are widely recognized as relevant in MBC susceptibility [20]. In our case series, $B R C A$ gene testing was carried out 
(according to the ASCO recommendations for BRCA testing [5]) on 43 subjects presenting at our department between January 2016 and December 2018. Among them, 17 were diagnosed with $\mathrm{MBC}$ and 26 were healthy highrisk men. Among the MBC patients, 2 presented with a $B R C A 2$ pathogenetic mutation (12\%). In our case series, $B R C A 2$ mutation carriers were older than wild-type $B R C A$ patients at diagnosis (median 78 vs. 65 years). This finding contradicts that of Deb et al. [20], who found a 10 -year-earlier onset of $\mathrm{MBC}$ in $B R C A 2$ mutation carriers than in wild-type $B R C A 1 / 2$ carriers. One possible explanation could be the extremely small number of MBC patients who were BRCA2 mutation carriers in our retrospective study, in addition to the extremely advanced age (90 years) of 1 patient. Figure 2 and Figure 3 show genealogical trees of our 2 cases of $\mathrm{MBC}$ in $B R C A 2$ mutation carriers.

Different studies have observed that $\mathrm{MBC}$ associated with $B R C A 2$ mutations presents with a more aggressive phenotype (higher histologic grade, greater Ki-67 expression, an advanced stage at diagnosis, and nodal involvement) than FBC associated with BRCA2 mutations and $\mathrm{MBC}$ with wild-type $B R C A[8,13,21]$. In our retrospective study, 2 cases of $\mathrm{MBC}$ in $B R C A 2$ mutation carriers were recorded. One was diagnosed as advanced-stage with high Ki-67 expression and extensive nodal involvement.

In $B R C A 1 / 2$ mutation carriers, a retrospective study indicated a higher risk before the age of 65 years of also developing PCa and pancreatic cancer [4]. Furthermore, studies have observed that $\mathrm{PCa}$ associated with BRCA2 mutations presents at a younger age, with more aggressive patterns (Gleason score $>8$, advanced stage $\mathrm{T}$, nodal involvement, and metastatic at diagnosis) than $\mathrm{PCa}$ in wild-type $B R C A$ [22-24]. In our retrospective study, only 5 patients were diagnosed with $\mathrm{PCa}$. This may be due to the relatively small sample size and the heterogeneity of the follow-up periods.

It is noteworthy that, according to our findings, a higher percentage of $B R C A$ mutation carriers were found among healthy men than MBC patients (50 vs. $11.8 \%$ ). This result is not surprising because we specifically selected high-risk patients for oncogenetic counseling, according to the ASCO recommendations [5]. In this way, we were able to obtain a $B R C A$ mutation study of some high-risk family clusters and involve these subjects in personalized screening programs including: clinical breast examination, mammography and contrast-enhanced MRI, clinical urologic examination, serum PSA level testing, and transrectal ultrasound for prostate evaluation.

This study has several limitations. Firstly, the relatively small sample size does not allow an absolute generalization of the observed results. Secondly, considerations regarding overall survival and disease-specific mortality were not possible due to the excessive heterogeneity of the follow-up periods. This study has as its strength the enrollment of healthy high-risk subjects who underwent BRCA genes mutation testing.

With this retrospective case series, we evaluated the distribution of clinicopathological characteristics in $\mathrm{MBC}$ subjects and assessed the frequency of mutations in $B R C A$ genes in affected patients and healthy high-risk subjects, with the aim of proposing a surveillance program for BC and PCa. Further investigations are clearly needed in order to evaluate the effectiveness of specifically designed screening programs for $\mathrm{MBC}$ and $\mathrm{PCa}$ in a larger population of healthy high-risk men.

\section{Statement of Ethics}

The authors have no ethical conflicts to disclose. The patients included in this retrospective analysis provided their informed consent in the framework of regular clinical activity of our institutions.

\section{Disclosure Statement}

The authors have no conflicts of interest to declare.

\section{Funding Sources}

All costs were handled by the hospital and the authors themselves. No funding from third parties was received.

\section{Author Contributions}

Francesca Pellini and Eleonora Granuzzo: conceptualization, investigation, data curation, writing (original draft), visualization, and supervision. Silvia Urbani: conceptualization, data curation, writing (review and editing), visualization, and supervision. Sara Mirandola, Elena Fiorio, Marta Mandarà, Davide Lombardi, Marina Caldana, and Giovanni Paolo Pollini: investigation, data curation, writing (review and editing) and visualization. All authors read and approved the final manuscript.

\footnotetext{
References 1 Javidiparsijani S, Rosen LE, Gattuso P. Male breast carcinoma: a clinical and pathological review. Int J Surg Pathol. 2017 May;25(3): 200-5.

2 Cardoso F, Bartlett JM, Slaets L, van Deurzen $\mathrm{CH}$, van Leeuwen-Stok E, Porter P, et al. Characterization of male breast cancer: results of the EORTC 10085/TBCRC/BIG/ NABCG International Male Breast Cancer Program. Ann Oncol. 2018 Feb;29(2):405-17.

3 Ly D, Forman D, Ferlay J, Brinton LA, Cook MB. An international comparison of male and female breast cancer incidence rates. Int $\mathrm{J}$ Cancer. 2013 Apr;132(8):1918-26.
} 
4 Ottini L, Palli D, Rizzo S, Federico M, Bazan V, Russo A. Male breast cancer. Crit Rev Oncol Hematol. 2010 Feb;73(2):141-55.

5 Korde LA, Zujewski JA, Kamin L, Giordano S, Domchek S, Anderson WF, et al. Multidisciplinary meeting on male breast cancer: summary and research recommendations. J Clin Oncol. 2010 Apr;28(12):2114-22.

6 Brewer HR, Jones ME, Schoemaker MJ, Ashworth A, Swerdlow AJ. Family history and risk of breast cancer: an analysis accounting for family structure. Breast Cancer Res Treat. 2017 Aug;165(1):193-200.

7 Hong JH, Ha KS, Jung YH, Won HS, An HJ, Lee GJ, et al. Clinical features of male breast cancer: experiences from seven institutions over 20 years. Cancer Res Treat. 2016 Oct; 48(4):1389-98.

8 Silvestri V, Barrowdale D, Mulligan AM, Neuhausen SL, Fox S, Karlan BY, et al.; kConFab Investigators; Hereditary Breast and Ovarian Cancer Research Group Netherlands (HEBON); EMBRACE. Male breast cancer in BRCA1 and BRCA2 mutation carriers: pathology data from the Consortium of Investigators of Modifiers of BRCA1/2. Breast Cancer Res. 2016 Feb;18(1):15

9 Castro E, Goh C, Olmos D, Saunders E, Leongamornlert D, Tymrakiewicz M, et al. Germline BRCA mutations are associated with higher risk of nodal involvement, distant metastasis, and poor survival outcomes in prostate cancer. J Clin Oncol. 2013 May;31(14): 1748-57.
10 Elston CW. Classification and grading of invasive breast carcinoma. Verh Dtsch Ges Pathol. 2005;89:35-44.

11 Senkus E, Kyriakides S, Ohno S, Penault-Llorca F, Poortmans P, Rutgers E, et al.; ESMO Guidelines Committee. Primary breast cancer: ESMO Clinical Practice Guidelines for diagnosis, treatment and follow-up. Ann Oncol. 2015 Sep;26(5 Suppl 5):v8-30.

12 http://research.nhgri.nih.gov/bic/

13 Gargiulo P, Pensabene M, Milano M, Arpino G, Giuliano M, Forestieri V, et al. Long-term survival and BRCA status in male breast cancer: a retrospective single-center analysis. BMC Cancer. 2016 Jul;16(1):375.

14 Masci G, Caruso M, Caruso F, Salvini P, Carnaghi $\mathrm{C}$, Giordano L, et al. Clinicopathological and immunohistochemical characteristics in male breast cancer: a retrospective case series. Oncologist. 2015 Jun;20(6):586-92.

15 Kornegoor R, Verschuur-Maes AH, Buerger $\mathrm{H}$, Hogenes MC, de Bruin PC, Oudejans JJ, et al. Molecular subtyping of male breast cancer by immunohistochemistry. Mod Pathol. 2012 Mar;25(3):398-404.

16 Zhou FF, Xia LP, Guo GF, Wang X, Yuan ZY, Zhang B, et al. Changes in therapeutic strategies in Chinese male patients with breast cancer: 40 years of experience in a single institute. Breast. 2010 Dec;19(6):450-5.

17 Flynn LW, Park J, Patil SM, Cody HS 3rd, Port ER. Sentinel lymph node biopsy is successful and accurate in male breast carcinoma. J Am Coll Surg. 2008 Apr;206(4):616-21.
18 Yu E, Suzuki H, Younus J, Elfiki T, Stitt L, Yau $\mathrm{G}$, et al. The impact of post-mastectomy radiation therapy on male breast cancer patients - a case series. Int J Radiat Oncol Biol Phys. 2012 Feb;82(2):696-700.

19 Giordano SH, Perkins GH, Broglio K, Garcia SG, Middleton LP, Buzdar AU, et al. Adjuvant systemic therapy for male breast carcinoma. Cancer. 2005 Dec;104(11):2359-64

20 Deb S, Lakhani SR, Ottini L, Fox SB. The cancer genetics and pathology of male breast cancer. Histopathology. 2016 Jan;68(1):110-8.

21 Ottini L, Silvestri V, Rizzolo P, Falchetti M, Zanna I, Saieva C, et al. Clinical and pathologic characteristics of BRCA-positive and BRCA-negative male breast cancer patients: results from a collaborative multicenter study in Italy. Breast Cancer Res Treat. 2012 Jul; 134(1):411-8.

22 Kote-Jarai Z, Leongamornlert D, Saunders E, Tymrakiewicz M, Castro E, Mahmud N, et al.; UKGPCS Collaborators. BRCA2 is a moderate penetrance gene contributing to youngonset prostate cancer: implications for genetic testing in prostate cancer patients. Br J Cancer. 2011 Oct;105(8):1230-4.

23 Bancroft EK, Page EC, Castro E, Lilja H, Vickers A, Sjoberg D, et al.; IMPACT Collaborators. Targeted prostate cancer screening in BRCA1 and BRCA2 mutation carriers: results from the initial screening round of the IMPACT study. Eur Urol. 2014 Sep;66(3):48999. 